\title{
A Case of a Beta-Human Chorionic Gonadotropin Secreting Sinonasal Teratocarcinosarcoma
}

\author{
Benjamin D. Weinberg ${ }^{1}$ Kathy L. Newell ${ }^{2}$ Fen Wang ${ }^{3}$ \\ ${ }^{1}$ Department of Radiation Oncology, Wayne State University, Detroit, \\ Michigan, United States \\ 2 Department of Pathology, University of Kansas Medical Center, \\ Kansas City, Kansas, United States \\ ${ }^{3}$ Department of Radiation Oncology, University of Kansas Medical \\ Address for correspondence Benjamin D. Weinberg, MD, MA, \\ Department of Radiation Oncology, Wayne State University, 4201 \\ St. Antoine Boulevard, 1D-UHC, Detroit, MI 48201, United States \\ (e-mail: med@bensbrain.com).
} Center, Kansas City, Kansas, United States

J Neurol Surg Rep 2014;75:e103-e107.

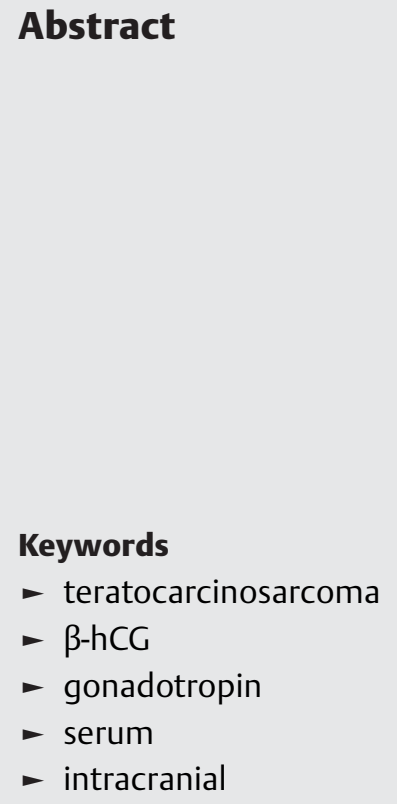

Background and Importance Sinonasal teratocarcinosarcoma (SNTCS) is an unusual and aggressive neoplasm characterized by the combination of teratoma and carcinosarcoma features that is often misdiagnosed based on a biopsy sample. Approximately 60 cases have been reported in the English literature, but none have been associated with serum tumor markers. We report a case of SNTCS with elevation of serum $\beta$-human chorionic gonadotropin (hCG) level.

Clinical Presentation The patient was a 22-year-old pregnant woman at diagnosis, and her $\beta$-hCG level was appropriately elevated. Her initial treatment was a surgical resection followed by radiation therapy (RT). Two years later, the patient had a pregnancy work-up after a sexual assault. Her serum $\beta$-hCG was elevated, but a nongravid uterus was seen on ultrasound. Magnetic resonance imaging (MRI) of the head revealed an intracranial tumor recurrence in the right frontal lobe. During salvage RT, $\beta$-hCG decreased rapidly to normal levels, with a complete tumor response on MRI. Retrospective examination of the initial surgical specimen showed expression of $\beta$-hCG associated with the anaplastic mesenchymal component.

Conclusion This is the first reported case of a $\beta$-hCG secreting SNTCS. The source of $\beta$-hCG production was traced to a neoplastic smooth muscle component of the tumor.

\section{Background and Importance}

Sinonasal teratocarcinosarcoma (SNTCS) is a very unusual and aggressive neoplasm first characterized as a separate entity by Heffner and Hyams in $1984 .^{1}$ It is currently defined by the World Health Organization as "a complex malignant sinonasal neoplasm combining features of teratoma and carcinosarcoma" that typically includes benign and malignant epithelial, mesenchymal, and neuroepithelial elements including immature tissue with blastomatous features. ${ }^{2}$ Unlike other malignant mixed germ cell tumors (GCTs), SNTCS lacks embryonal carcinoma, yolk sac tumor, choriocarcinoma, and seminoma components. Based on an inadequate sample, this heterogenous tumor can be misdiagnosed as olfactory neuroblastoma, squamous cell carcinoma, undifferentiated carcinoma, adenocarcinoma, malignant salivary gland-type tumors, or adenosquamous carcinoma. ${ }^{2}$ A recent comprehensive review of the literature by Wei et al revealed $<60$ cases reported in the English literature. ${ }^{3}$ The primary treatment in $82 \%$ of the cases reviewed was surgery, with or without adjuvant radiation therapy (RT). The remainder received definitive RT. Many patients in both groups received some form of chemotherapy as well. There were high rates of recurrence and metastasis overall, although no clear received

August 24, 2013

accepted

February 10, 2014

published online

May 2, 2014
DOI http://dx.doi.org/

10.1055/s-0034-1373667. ISSN 2193-6358. (c) 2014 Georg Thieme Verlag KG
Stuttgart · New York

License terms

((1) $\circledast \circledast$ 


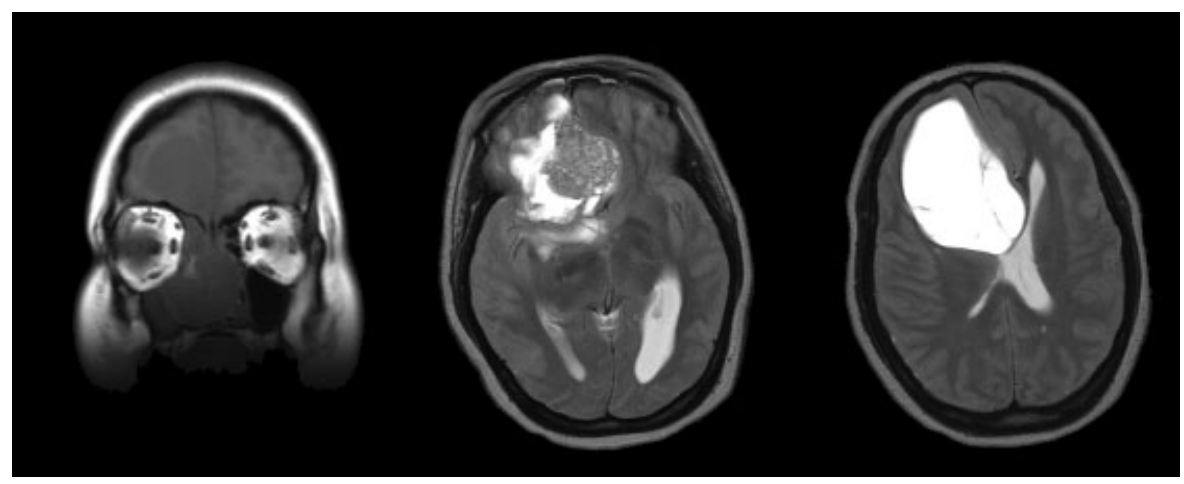

Fig. 1 Noncontrast magnetic resonance imaging of the head showing a large cystic mass in the paranasal sinuses and frontal lobe.

prognostic factors were identified. Overall, 1-year and 3-year survival rates were $36 \%$ and $25 \%$, respectively, for those patients with reported outcomes. ${ }^{3}$ Of all the reported cases of SNTCS, only one has shown any hormone secretion: antidiuretic hormone, causing hyponatremia. ${ }^{4}$ In that case, sodium levels were a reliable marker of tumor control and were an early postoperative indicator of tumor recurrence. To date, no SNTCS with elevated GCT markers has been reported. We present a case in which a highly radiosensitive SNTCS was associated with elevated $\beta$-hCG.

\section{Clinical Presentation}

A 22-year-old African American woman presented to the emergency department at $\sim 16$ weeks of gestation with a 2- to 3-month history of worsening headaches and a 3-week history of increasing somnolence, nausea, vomiting, weight loss, and personality changes.

A magnetic resonance imaging (MRI) of her head revealed an $11-\mathrm{cm}$ right frontal mass with a 7-cm cystic component causing significant compression of the right frontal and temporal lobes, mild herniation of the right uncus, and mild hydrocephalus. The tumor extended through the anterior cranial base to the nasal cavity and paranasal sinuses (-Fig. 1).

On day 2 of admission, she had rapid mental status decline and underwent an emergent decompressive craniotomy. The tumor was extra-axial, and the cyst expressed a dark fluid "similar to crankcase oil." The small biopsy specimen taken during this procedure revealed neoplastic cells with squamous and basaloid differentiation and necrosis, leading to the impression of basaloid squamous cell carcinoma.

She subsequently underwent a combined endoscopic transnasal and cranial resection of the entire anterior cranial fossa and nasal cavity mass on day 28 of admission. The surgical specimen consisted of a $9.0 \times 8.5 \times 3.5-\mathrm{cm}$ aggregate of tan-brown soft tissue and bony fragments. Histologically, the tumor consisted of a heterogeneous mixture of malignant epithelial, mesenchymal, and primitive neuroepithelial elements. Tumor infiltration of brain tissue was observed. The epithelial component included squamous and basaloid carcinoma features (-Fig. 2). The mesenchymal component included areas of hypercellular smooth muscle, ranging from mildly anaplastic to overtly malignant, with focal areas of osteoid deposition ( - Fig. 3). The neuroepithelial component included neuroblastoma-like and primitive neuroectodermal tumor-like areas (-Fig. 4). Immunohistochemical staining revealed expression of vimentin, pancytokeratin, and 34ßE12, with focal positivity for CD99 and synaptophysin among the heterogeneous tumor components. After internal and external review, a diagnosis of sinonasal teratocarcinosarcoma was made.

Postoperative RT was recommended after surgery but was delayed for 5 months due to a complicated hospital course and family concerns about her pregnancy. The radiation planning MRI showed some residual soft tissue thickening

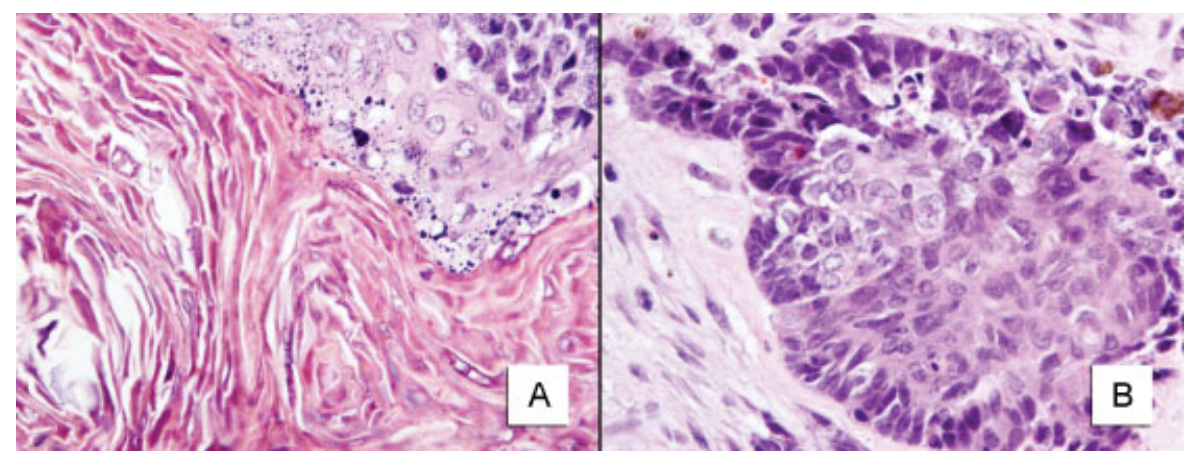

Fig. 2 Epithelial component. (A) Squamous epithelium (hematoxylin and eosin [H\&E]). (B) Basaloid epithelium (H\&E). 


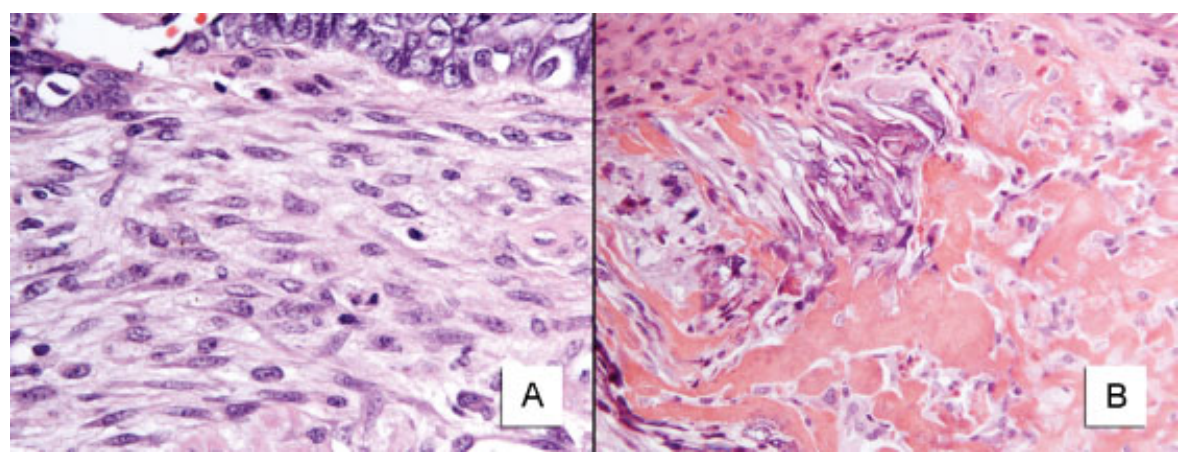

Fig. 3 Mesenchymal component. (A) Stromal smooth muscle with mitoses (hematoxylin and eosin [H\&E]). (B) Osteoid (lower right) admixed with epithelial component (upper left) (H\&E).

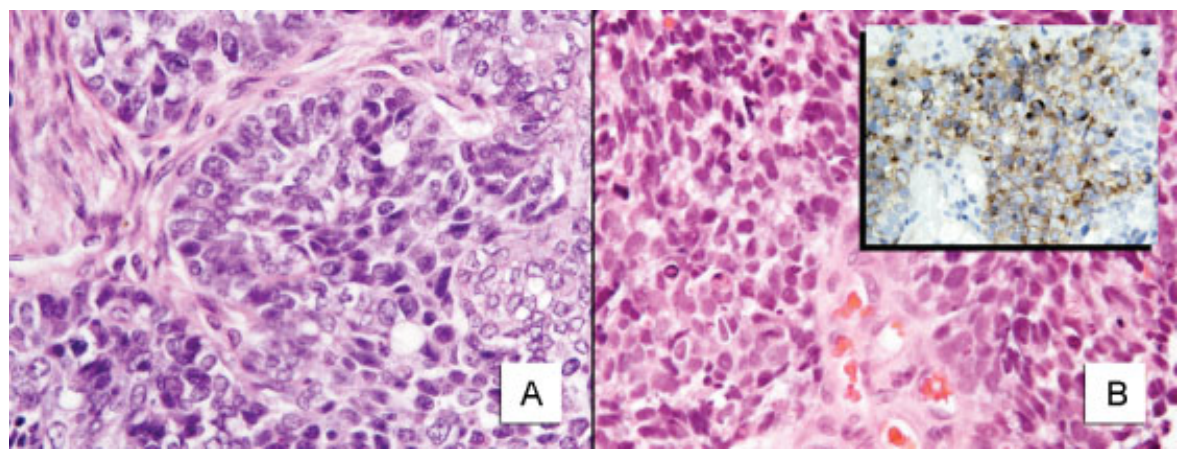

Fig. 4 Neuroectodermal component. (A) Neuroblastoma-like area. (B) Primitive neuroectodermal tumor-like area; hematoxylin and eosin; synaptophysin (inset).

and gyral enhancement in the floor of the craniotomy site but no gross tumor. After delivery of a healthy infant, she underwent a 2-month course of RT to the surgical bed to a total dose of $60 \mathrm{~Gy}$. The follow-up MRIs showed no evidence of recurrent disease for 18 months post-RT.

Two years (27 months) after finishing her postoperative $\mathrm{RT}$, she was admitted for evaluation through the emergency department following an alleged sexual assault by a family member. Her quantitative serum $\beta$-human chorionic gonadotropin (hCG) was elevated (60 U/L; normal nonpregnant range: $0-3$ ) but did not rise as would be expected for pregnancy. Pelvic and transvaginal ultrasound studies showed a normal-appearing nongravid uterus. MRI of the head showed a recurrent intracranial mass in the right frontal lobe ( - Fig. 5A). She was deemed a high surgical risk, so reirradiation of the tumor was recommended. Serum $\beta$-hCG was monitored throughout the admission. Radiation was delayed by 45 days, and the planning MRI showed significant interval growth of the tumor with further elevation of $\beta$-hCG to $130 \mathrm{U} / \mathrm{L}$ (- Fig. 5B), but there were no new masses in the brain.

Because of clinical concern over the origin of the serum $\beta$-hCG elevation, the previous tumor tissue was examined with additional immunohistochemical staining for smooth muscle actin and $\beta$-hCG expression. $\beta$-hCG was found in areas of anaplastic smooth muscle (-Fig. 6 ).

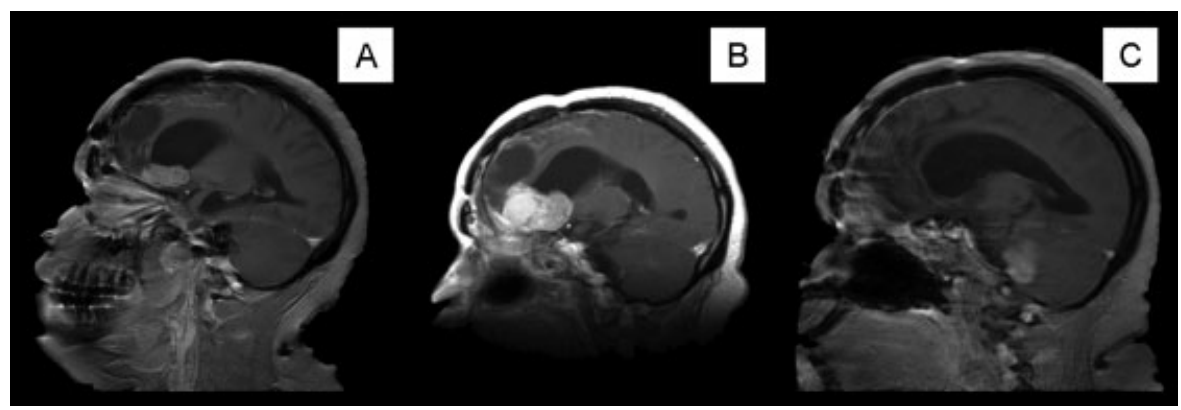

Fig. 5 (A) Magnetic resonance imaging (MRI) showing frontal lobe recurrence 27 months after radiation therapy. (B) MRI showing tumor growth 45 days later, before reirradiation. (C) MRI showing complete response of frontal lobe mass (and new posterior fossa disease) 1 month after reirradiation. 


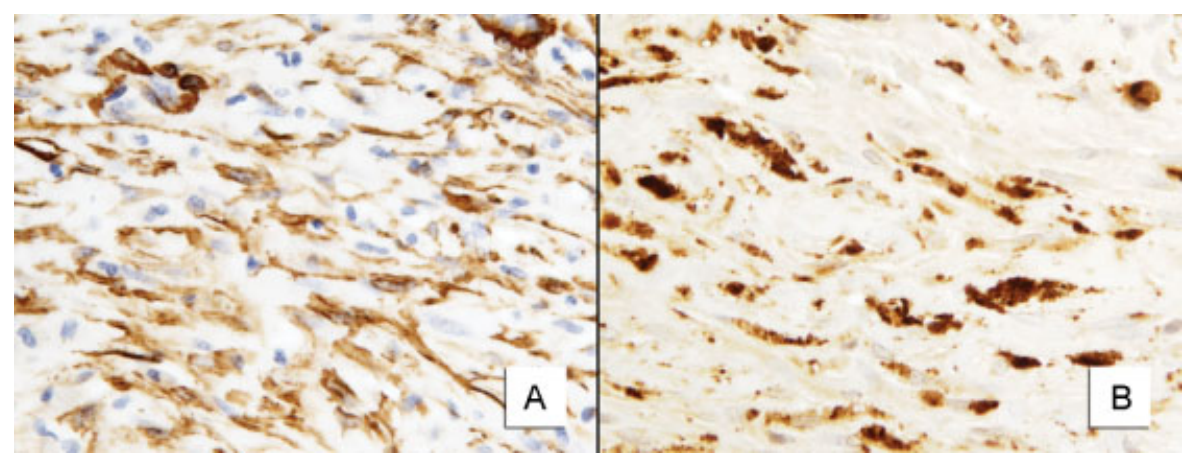

Fig. 6 Smooth muscle component. (A) Smooth muscle focus; smooth muscle actin. (B) $\beta$-human chorionic gonadotropin.

The patient received $48 \mathrm{~Gy}$ (of a planned $50 \mathrm{~Gy}$ ) of RT to the recurrent mass in 24 fractions. She missed many appointments but finished most of her prescribed course in a 6-week period with no acute morbidity. Computed tomography scan after the 10th fraction showed significant tumor response, and the treatment was replanned to account for the smaller tumor volume. Her serum $\beta$-hCG level dropped from a peak of $130 \mathrm{U} / \mathrm{L}$, measured at the start of RT, to $6 \mathrm{U} / \mathrm{L}$ (almost within normal limits) at the end of treatment ( - Fig. 7 ),

One month later she was admitted to the hospital again for increasing ataxia and falls. A brain MRI showed resolution of the enhancing frontal lobe mass (-Fig. 5C) but revealed at least five intracranial masses in the posterior fossa, outside of the prior RT fields. Serum $\beta$-hCG level was in the normal range. The decision was made to palliate with $30 \mathrm{~Gy}$ of RT to the posterior fossa in 10 fractions. There was no biopsy or follow-up imaging. She was discharged to hospice and died a month later. An autopsy was not performed.

\section{Discussion}

By definition, SNTCS do not contain areas of embryonal carcinoma, choriocarcinoma, yolk -sac tumor, or seminoma. They are hypothesized to arise from primitive pluripotent olfactory epithelial cells rather than germ cells. Thus they are traditionally believed to be incapable of producing GCT markers including $\beta$-hCG or $\alpha$-fetoprotein, although they have rarely been noted to produce pituitary hormones. ${ }^{4}$

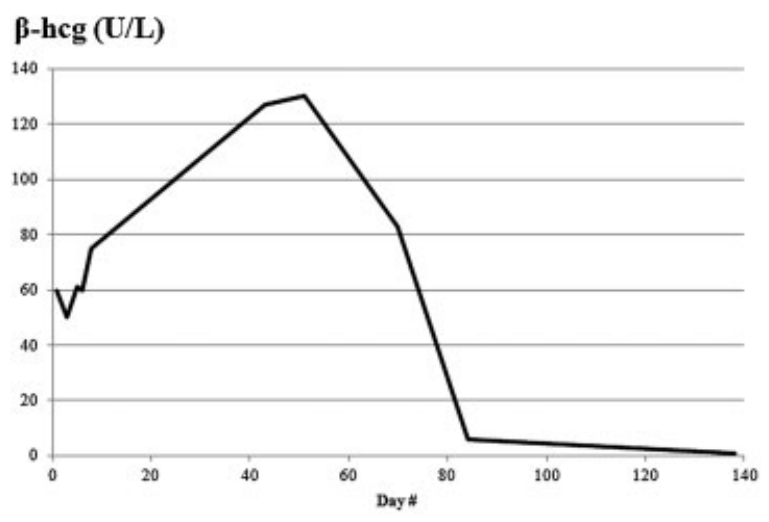

Fig. 7 Serum $\beta$-human chorionic gonadotropin levels before and after radiation therapy (RT) for recurrence in frontal lobe. RT was started on day 45.
These tumors comprise varying combinations of epithelial, mesenchymal, and neuroectodermal components exhibiting varying maturation. The mesenchymal component may appear along a continuum from benign to malignant and can include mature components such as cartilage, bone, and striated or smooth muscle. Malignant sarcomatous transformation is also documented. ${ }^{1,5-7}$

In this case, smooth muscle actin highlighted an anaplastic smooth muscle component in this heterogeneous tumor. There are rare reports of pure leiomyosarcomas secreting $\beta$-hCG in the literature. ${ }^{8-10}$ In the absence of the GCT components just mentioned, the neoplastic smooth muscle component of the tumor with expression of $\beta$-hCG was the likely source for the elevated serum $\beta$-hCG levels.

Because this patient was pregnant on initial presentation, any tumor-related $\beta$-hCG production at that time was masked by the elevated serum levels associated with pregnancy. However, on recurrence, tumor production of $\beta$-hCG was confirmed with elevation of serum $\beta$-hCG, expression of $\beta$-hCG on the smooth muscle component of the tumor tissue, and correlation of serum $\beta$-hCG levels with changes in tumor size seen on imaging.

This patient's recurrent tumor initially showed a complete response to RT by both serum marker and imaging criteria. Subsequent tumor growth outside of the RT field was not associated with elevation of serum $\beta$-hCG.

\section{Conclusion}

SNTCS is a rare and unusual neoplasm that lacks embryonal carcinoma, yolk sac tumor, choriocarcinoma, and seminoma components. This is the first reported case of a $\beta$-hCG secreting SNTCS. The source of $\beta$-hCG production was traced to a neoplastic smooth muscle component of the tumor. Serum levels of this hormone were a reliable tumor marker during the patient's first recurrence and subsequent RT.

\section{Acknowledgments}

The authors wish to acknowledge the following individuals for their contributions in evaluating this complex tumor: Dr. Pratima Singh and Dr. Louis P. Dehner for diagnostic expertise, and Melissa Hull for immunohistochemical expertise. 


\section{References}

1 Heffner DK, Hyams VJ. Teratocarcinosarcoma (malignant teratoma?) of the nasal cavity and paranasal sinuses A clinicopathologic study of 20 cases Cancer 1984;53(10):2140-2154

2 Cardesa A, Luna MA. Germ cell tumours. In: Barnes L, Eveson JW, Reichart P, Sidransky D, eds. World Health Organization Classification of Tumours: Pathology \& Genetics-Head and Neck Tumours. Lyon, France: IARC; 2005:76-77

3 Wei S, Carroll W, Lazenby A, Bell W, Lopez R, Said-Al-Naief N. Sinonasal teratocarcinosarcoma: report of a case with review of literature and treatment outcome. Ann Diagn Pathol 2008;12(6): 415-425

4 Kleinschmidt-DeMasters BK, Pflaumer SM, Mulgrew TD, Lillehei KO. Sinonasal teratocarcinosarcoma ("mixed olfactory neuroblastoma-craniopharyngioma") presenting with syndrome of inappropriate secretion of antidiuretic hormone. Clin Neuropathol 2000;19(2):63-69

5 Kane SV, Karpate AA, Bal M, Juvekar SL, Pai PS. Chemotherapyinduced neuronal maturation in sinonasal teratocarcinosar- coma-a unique observation. Head Neck Pathol 2009;3(1): 31-36

6 Pai SA, Naresh KN, Masih K, Ramarao C, Borges AM. Teratocarcinosarcoma of the paranasal sinuses: a clinicopathologic and immunohistochemical study. Hum Pathol 1998;29(7):718-722

7 Shemen L, Galantich P, Murali R. Malignant teratocarcinosarcoma of the sphenoid sinus. Otolaryngol Head Neck Surg 1995;112(3): 496-500

8 Liang S, Stone G, Chalas E, Pearl M, Callan F, Zheng W. A high-grade uterine leiomyosarcoma with human chorionic gonadotropin production. Int J Gynecol Pathol 2006;25(3):257-261

9 Kelley BC, Arnold PM, Grant JA, Newell KL. Primary intracranial $\beta$-human chorionic gonadotropin-producing leiomyosarcoma in a 2-year-old immunocompetent child. J Neurosurg Pediatr 2012; 10(2):121-125

10 Froehner M, Gaertner HJ, Manseck A, Oehlschlaeger S, Wirth MP. Retroperitoneal leiomyosarcoma associated with an elevated betaHCG serum level mimicking extragonadal germ cell tumor. Sarcoma 2000;4(4):179-181 